\title{
Study on Pre-Deceleration Running in Encounters with Emergency Vehicles Using Vehicle to Vehicle Communications
}

\author{
Hideaki Nanba ${ }^{1}$ (D) $\cdot$ Manabu Sawada $^{2} \cdot$ Koji Oguri $^{3}$ \\ Received: 30 May 2019 / Revised: 12 September 2019 / Accepted: 4 November 2019 / Published online: 2 December 2019 \\ (C) The Author(s) 2019
}

\begin{abstract}
There is a problem of coordination in encounters with an emergency vehicle when an automated driving vehicle travels on public roads. Effectiveness of the pre-deceleration running based on the earlier recognition of emergency vehicle using vehicle to vehicle communication is studied from the viewpoint of safety and ride comfort. Experimental data of the driver's maneuver and vehicle's status are collected and analyzed using the driving simulator. Recognition by vehicle to vehicle communication (V2V for short) leads to pre-deceleration and improves deceleration and jerk as compared to siren sound and red light of emergency vehicles.
\end{abstract}

Keywords Pre-deceleration $\cdot$ Automated Driving $\cdot$ V2V $\cdot$ Emergency vehicle $\cdot$ Evacuation $\cdot$ ride comfort

\section{Introduction}

Technological development is expected in which automated driving vehicles run on public roads. When an automated driving vehicle travels on general road, unlike expressways and dedicated roads for automobiles, coexistence or coordination with a priority vehicle (such as an emergency vehicle) must be considered. And also, safety and less stress must be achieved for passengers of automated driving vehicles. Relating to this theme, the studies of vehicle to vehicle communications have been conducted [1]. In previous researches, the superiority of vehicle to vehicle communications is examined from the viewpoint of emergency vehicles. For example, shortening the travel time of the emergency vehicle thanks for

Hideaki Nanba

h.namba@bme.ist.aichi-pu.ac.jp

Manabu Sawada

manabu.sawada.j6j@jp.denso.com

Koji Oguri

oguri@ ist.aichi-pu.ac.jp

1 Graduate school of Information Science and Technology, Aichi Prefectural University, Nagakute, Japan

2 M Systems R\&D Department, Advanced Mobility Systems R\&D Division, Denso Corporation, Kariya, Japan

3 Institute of Information Science and Technology, Aichi Prefectural University, Nagakute, Japan the evacuating operation of surrounding vehicles by the early recognizing the emergency vehicle approach. However, the problems occurred on the surrounding vehicles are not examined. It is necessary that the stress of passenger should be reduced when automated driving vehicle becomes widespread in the future. This paper investigates any problem whether pre-deceleration is effective for safety and ride comfort.

\section{Superiority of V2V Communication in Emergency Vehicle Recognition}

The authors compared siren, red light, and vehicle to vehicle communication with regard to the distance at which the surrounding vehicles can recognize the approach of an emergency vehicle such as an ambulance.

The vehicle to vehicle communication uses a radio system (ARIB STD T-109) [2] that has been put to practical use in Japan in $760 \mathrm{MHz}$ band.

The distance of ambulance recognition is siren sound (average $228 \mathrm{~m}$ ), red light (average $217 \mathrm{~m}$ ), vehicle to vehicle communication (average $526 \mathrm{~m}$ ) in LOS (Line Of Sight), siren sound (average $168 \mathrm{~m}$ ), red light (average $36 \mathrm{~m}$ ) and vehicle to vehicle communication (average $278 \mathrm{~m}$ ) is reported in NLOS (Non Line Of Sight) [3]. Table 1 shows the recognized distance in the real situation.

Recognition of red light is the case where it can be recognized in spites of being many obstacles that obstruct the view. As for siren sound, the recognition depends on the quietness in 
Table 1 The recognized distance in the real situation

\begin{tabular}{lllll}
\hline & & Red light & Siren & V2V \\
\hline \multirow{2}{*}{ LOS } & Max. & $374 \mathrm{~m}$ & $380 \mathrm{~m}$ & $570 \mathrm{~m}$ \\
& Min. & $137 \mathrm{~m}$ & $107 \mathrm{~m}$ & $414 \mathrm{~m}$ \\
& Ave. & $217 \mathrm{~m}$ & $228 \mathrm{~m}$ & $526 \mathrm{~m}$ \\
\multirow{2}{*}{ NLOS } & Max. & $49 \mathrm{~m}$ & $233 \mathrm{~m}$ & $302 \mathrm{~m}$ \\
& Min. & $29 \mathrm{~m}$ & $70 \mathrm{~m}$ & $196 \mathrm{~m}$ \\
& Ave. & $36 \mathrm{~m}$ & $168 \mathrm{~m}$ & $287 \mathrm{~m}$ \\
\hline
\end{tabular}

the vehicle interior or driver's music listening. As for the communicable distance of vehicle to vehicle communication, Automobile Bureau of MLIT (Ministry of Land, Infrastructure, Transport and Tourism) compiled the guidelines of the communication operation type driving support system in 2011 [1]. It stated that the information is started providing when the linear distance with the emergency vehicle becomes within $300 \mathrm{~m}$.

In the real environment, the recognizable distance fluctuates depending on the environmental condition (e.g. radio wave shielding building). When siren sound, red light, and vehicle to vehicle communication are compared at the recognition distance reported in the literature [3], the vehicle to vehicle communication has an advantage in early recognition. It is confirmed that the ambulance approach could be recognized by the vehicle to vehicle communication before the time when the siren sound is heard even in NLOS environment. In this way, vehicle to vehicle communication is shown to be superior to emergency vehicle recognition (herein, emergency vehicle is referred to as ambulance in this paper), but it is unknown about how the driver takes advantage of the merit and uses it. It is not able to be grasped without observation.

However, the chance of actually encountering an ambulance on public roads is extremely rare and it is not practical to conduct measurement experiments in a real environment. Therefore, authors decide to collect data by experiments using a driving simulator with specific scenarios.

\section{Experiments by Driving Simulator}

\subsection{Driving Simulator System}

The driving simulator used is manufactured by Forum Eight Co., Ltd. The vehicle body is equipped with a 6-axis motion platform, and the seat moves in conjunction with accelerator, brake and steering operations. The screen has a $26-$ in. LCD monitor on the left, right and front. 7-in. display device with navigation and ambulance approach notification function is on the left side of the driver's seat.

As far as the construction of the virtual road environment, the virtual reality creation software "UC-win / Road Ver. 10
Driving Sim" for the driving simulator of FORUM8 is applied. In UC-win / Road Ver. 10 Driving Sim, the roadways, sidewalks, roadside buildings, roadside trees, traffic lights, etc. are placed in a virtual space to represent an environment close to the actual road environment. The ambulance approaching from backward is recognized by confirming the image on the rear mirror. Ambulance travel is also realized with this function.

Figure 1 shows the appearance of the driving simulator, and Fig. 2 shows the system configuration of the driving simulator.

Figure 3 shows the front screen and the navigation display screen of the driving simulator. An image of ambulance approaching is displayed on the navigation display screen.

\subsection{Subject}

When conducting an experiment using a driving simulator for understanding the characteristics of a human driver, it is suitable for the subject to drive daily.

Authors also think of eliminating factors that bias the experimental results. Candidates of the subjects are 75 candidates selected from the generally recruited candidates by screening survey. The condition of the screening is "I have a driver's license and drive one or more times a week". As a result, the frequency of driving is $50 \%, 32 \%$, and $17 \%$ for those who operate 1 to 2 times, 3 to 5 times, and 6 times or more a week. The distribution of gender and age of the subjects is almost the same as $49 \%$ women and $51 \%$ men. The age distribution is almost equally distributed to $30 \mathrm{~s} 33 \%, 40 \mathrm{~s}$ $33 \%$ and 50 s $34 \%$.

The experiment was conducted in Tokyo from December 1 to 24,2015 after necessary procedures based on respect for human rights.

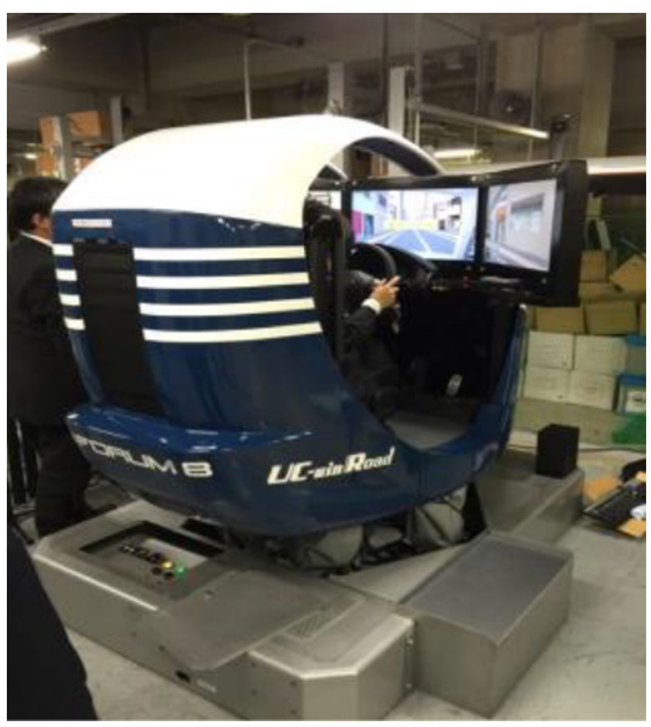

Fig. 1 Appearance of the driving simulator 


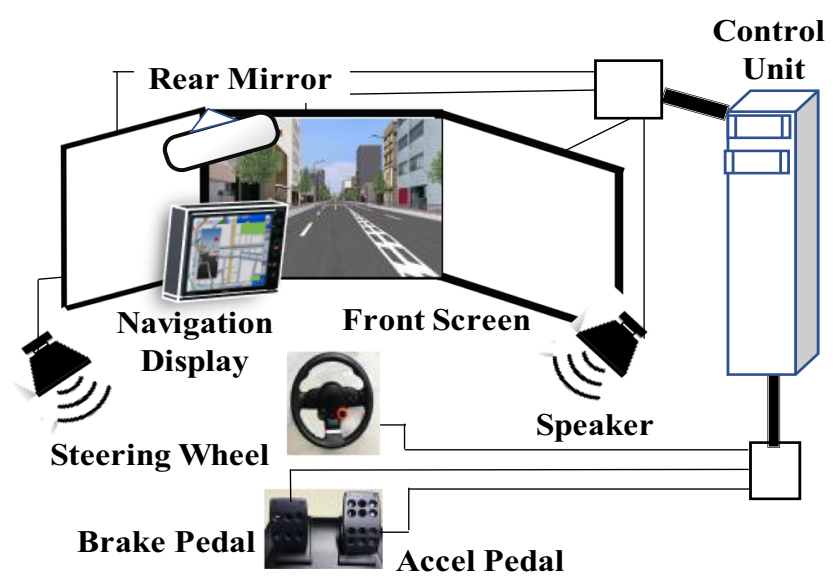

Fig. 2 Driving simulator system configuration

\subsection{Experimental Process}

The driving scene of the subject in the driving simulator is taken with a video camera from two places in front of and behind the driver's seat, and the video is recorded. An interview survey is conducted after the experiment of the driving simulator. The recorded video is used to confirm the time of triggers related to recognition and evasion when the ambulance approaches. With regard to recognized time and avoidance start time, accurate identification in interviews is difficult, and for reference, the accelerator and brake signals are actually extracted from the log data of the experiment to estimate the time. The experimental scenario is set as follows. The siren sound is pronounced when approaching to a distance of $60 \mathrm{~m}$.

According to the report by Baba et al. [4], about half of drivers notice the sound in the distance to the ambulance being about $60 \mathrm{~m}$, and so the threshold is set to a value of $60 \mathrm{~m}$ in the

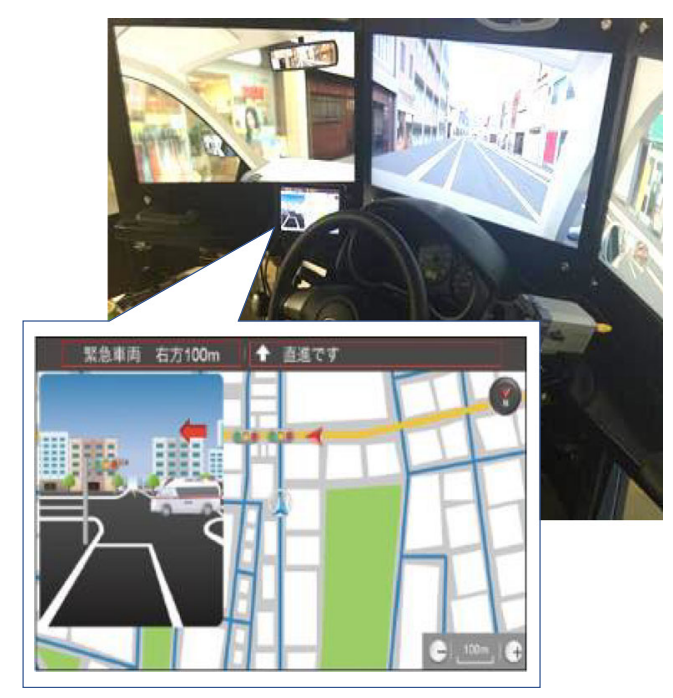

Fig. 3 Forward screen and car-navigation display experiment. It is set so that the volume of the sirens would increase every $20 \mathrm{~m}$. The red light is displayed on the rear mirror when there is nothing to obstruct the view when the ambulance approaches from behind. As for vehicle to vehicle communication, the notification of the approach of the ambulance is displayed on the navigation screen with a warning sound of "Pon".

When notifying by vehicle to vehicle communication, the time to notify is when the distance from the ambulance becomes within about $300 \mathrm{~m}$ in the case of backward approach. $100 \mathrm{~m}$ from the intersection where the ambulance encounters is in the case of lateral direction approach. It is taken into consideration of the attenuation due to radio wave shielding of the surrounding environment. Also, when the ambulance approaches the target vehicle (subject driving vehicle) up to $100 \mathrm{~m}$, the characters " $100 \mathrm{~m}$ approach" appears on the navigation display.

The scenario is configured by mixing cases with and without vehicle to vehicle communication for comparison. In addition, 7 scenarios without encountering the ambulance are prepared as a dummy, because the subject would pose if he informed that the ambulance approached in advance. One subject is given a trial run for a total of 10 scenarios with 3 scenarios including an ambulance approach scenario.

Figure 4 shows an example of scenario setting per subject.

\subsection{Driving Scenario from Backward Approach}

Figure 5 shows an experimental scheme in which the subject drives the target vehicle and the ambulance

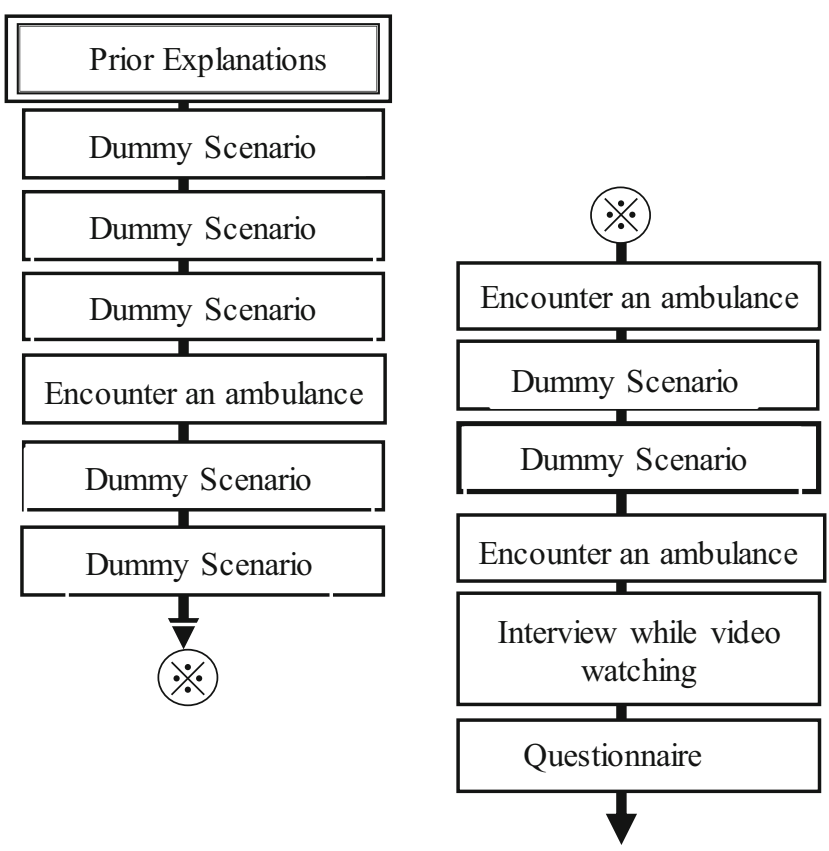

Fig. 4 Scenario configuration of a subject 


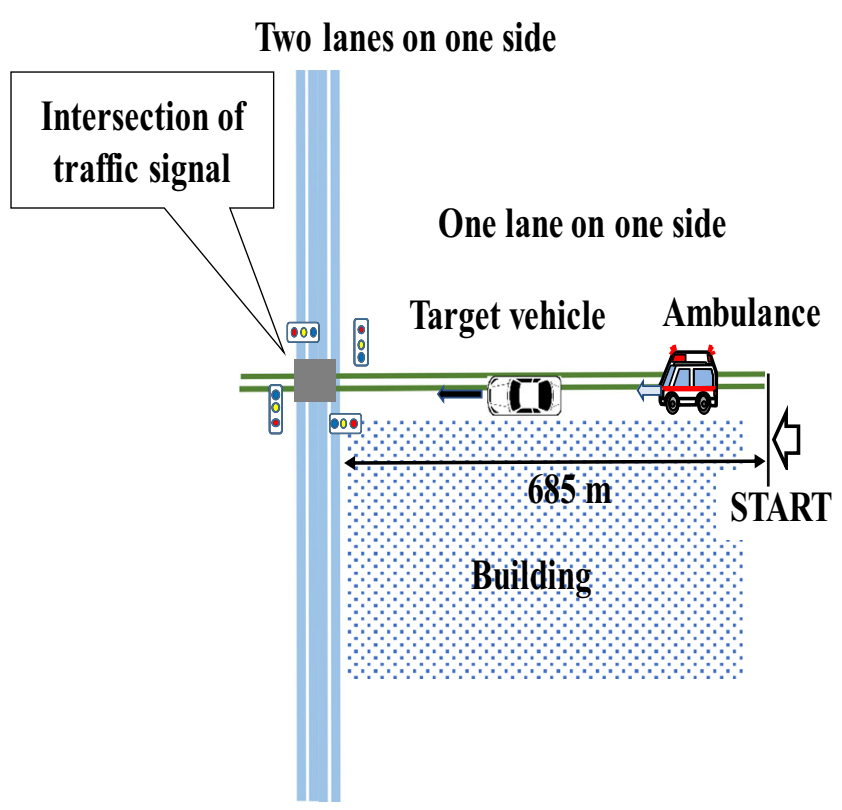

Fig. 5 Driving scenario of an ambulance from backward approach

approaches from backward. The target vehicle travels a road with one lane on one side, there is an intersection ahead, and the target vehicle moves away from the side of the road and stops to take an evasive action to give way to the emergency vehicle.

The amount of operation of each subject while driving and the status of the vehicle are recorded every $0.1 \mathrm{~s}$.

The recording target is the data indicating the status of the target vehicle, such as the subject's ID, accelerator depression rate, brake depression rate, steering wheel operation angle, vehicle type, position (latitude, longitude, altitude), velocity, etc. There are 81 types of records. The time when the subject started traveling from the position

\section{Two lanes on one side}

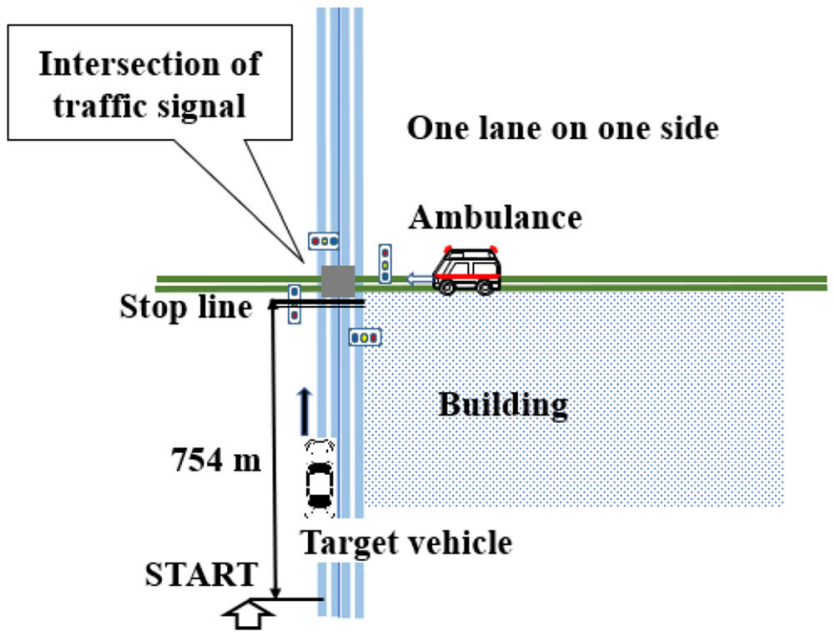

Fig. 6 Driving scenario of an ambulance from lateral direction approach of START is set to $0.0 \mathrm{~s}$, and the elapsed time is recorded as a time axis in units of $0.1 \mathrm{~s}$.

\subsection{Driving Scenario from lateral direction approach}

Figure 6 shows an experimental scheme in which the subject drives the target vehicle and the ambulance approaches from lateral direction. The target vehicle travels a road with two lanes on one side, and there is an intersection ahead. In the surrounding area, radio environments and outlook are bad. Even if the forward traffic signal is "green", the target vehicle decelerates and stops and takes evasive action to give way to the ambulance.

The time is the same as in the backward approach, and the time when the subject started traveling from the START position is $0.0 \mathrm{~s}$, and the elapsed time is recorded as a time axis in units of $0.1 \mathrm{~s}$. Moreover, the recording object is the same as in the case of the backward approaching.

\section{Experimental Data Using Non-V2V Recognition}

\subsection{The Case of the Siren Sound Recognition from Backward Approach}

There are ten subjects who experimented with a scenario of recognizing with a siren sound when an ambulance approached from backward. Table 2 shows experimental data of five persons excluding extreme driving operation data (for example, without stopping and applying an emergency brake) according to the individuality of the subject. Figure 7 shows the experimental data No.63_7_1B, which has the shortest time to stop among the subjects. Figure 7 shows the data of the brake depression amount, velocity, deceleration and jerk. At the time of recognition, the distance between the target vehicle and the ambulance is $57.9 \mathrm{~m}$, and the velocity of the target vehicle is approximately $32.2 \mathrm{~km} / \mathrm{h}$. Awareness is defined as the time when a subject recognizes an ambulance approach.

In this case, the subject recognizes the ambulance's approach with a siren sound at $58.8 \mathrm{~s}$. After recognition, he starts applying the brake and stops at $64.1 \mathrm{~s}$ and gives way to the ambulance.

A maximum deceleration of $-4.24 \mathrm{~m} / \mathrm{s}^{2}$ and a jerk of $-0.73 \mathrm{~m} / \mathrm{s}^{3}$ have occurred until the target vehicle stops. The measured data usually contains complex noise. In this experiment, the deceleration is expressed only by subtracting the vehicle velocity, but the jerk indicates the noise removed by the Butterworth filter. 
Table 2 The data of five subjects notifying an ambulance from backward by siren sound

\begin{tabular}{|c|c|c|c|c|c|c|}
\hline & Data No. & $\begin{array}{l}\text { Velocity at } \\
\text { recognition }[\mathrm{km} / \mathrm{h}]\end{array}$ & $\begin{array}{l}\text { Distance at } \\
\text { recognition }[\mathrm{m}]\end{array}$ & $\begin{array}{l}\text { Time before } \\
\text { stop [sec] }\end{array}$ & $\begin{array}{l}\text { Maximum } \\
\text { declaration }\left[\mathrm{m} / \mathrm{s}^{2}\right]\end{array}$ & $\begin{array}{l}\operatorname{Maximum} \\
\operatorname{jerk}\left[\mathrm{m} / \mathrm{s}^{3}\right]\end{array}$ \\
\hline \multirow{6}{*}{$\begin{array}{l}\text { Backward Approach/ } \\
\text { Siren Sound } \\
\text { Awareness }\end{array}$} & 63_7_1B & 32.2 & 57.9 & 5.3 & -4.24 & -.0 .73 \\
\hline & 40_4_1B & 28.7 & 59.1 & 16.5 & -2.44 & -.0 .63 \\
\hline & $54 \_4 \_1 \mathrm{~B}$ & 29.1 & 53.5 & 6.8 & -2.29 & -0.61 \\
\hline & $45_{1}{ }_{10}-$ & 32.7 & 55.1 & 8.1 & -2.26 & -0.6 \\
\hline & $67+7 \_1 \mathrm{E}$ & 29 & 56.7 & 8.4 & -2.81 & -0.57 \\
\hline & Average & 30.34 & 56.46 & 9.02 & -2.808 & -0.626 \\
\hline
\end{tabular}

The Butterworth filter used is described in Chapter 5. The average data of five subjects is shown in Table 3.

\subsection{The Case of the Red Light Recognition from Backward Approach}

There are ten subjects who experimented with a scenario of recognizing with a red light when an ambulance approached from backward. Five subjects excluding extreme driving operation data (for example, without stopping and applying an emergency brake) are considered. The average data of five persons is shown in Table 3. Figure 8 shows the experimental

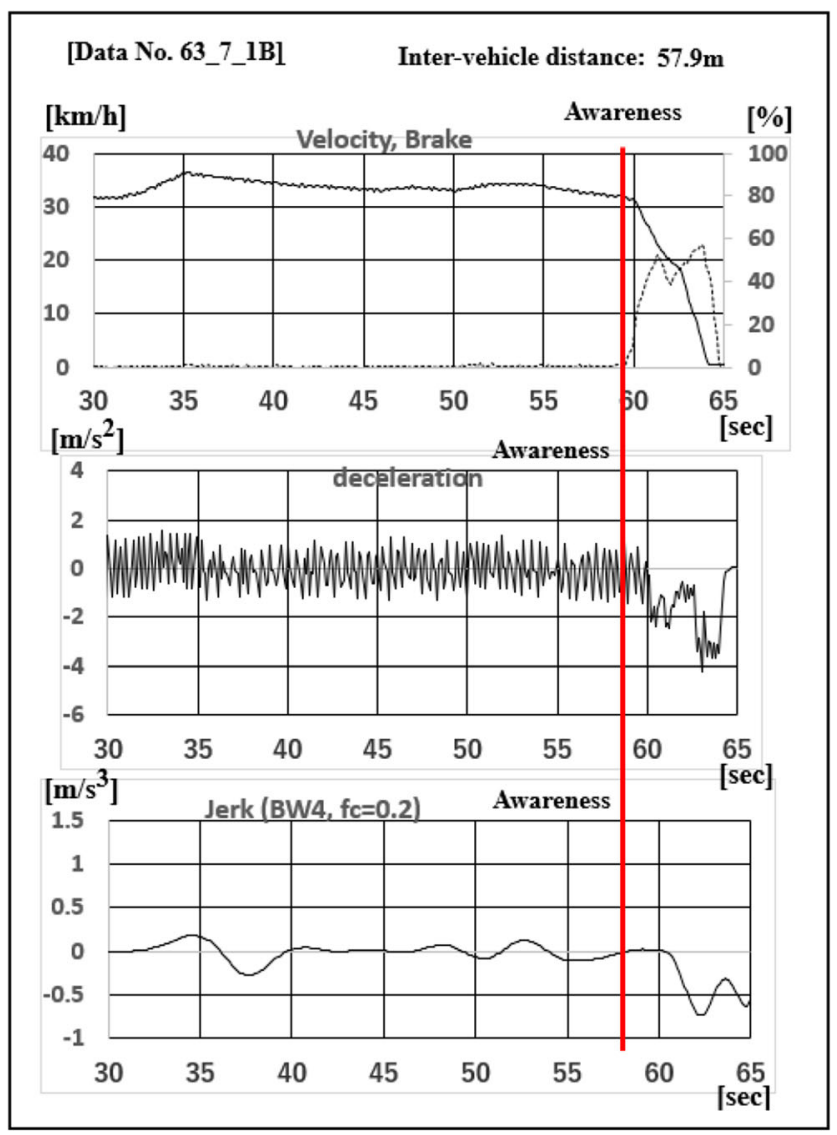

Fig. 7 Velocity \& Brake, Deceleration and Jerk in Notice by siren sound from backward data No $.58 \_4 \_$E, which is near to the average data. Figure 8 shows the brake depression amount, velocity, deceleration and jerk. At the time of recognition, the distance between the target vehicle and the ambulance is $54.3 \mathrm{~m}$, and the velocity of the target vehicle is approximately $26.6 \mathrm{~km} / \mathrm{h}$. The subject recognizes the approach of the ambulance with the red light reflected on the rearview mirror at $53.3 \mathrm{~s}$. After recognition, he starts applying the brake and stops at $60.6 \mathrm{~s}$ and gives way to the ambulance. A maximum deceleration of $-3.09 \mathrm{~m} / \mathrm{s}^{2}$ and a jerk of $-0.895 \mathrm{~m} / \mathrm{s}^{3}$ have occurred until the target vehicle stops.

\subsection{The Case of the Siren Sound Recognition from Lateral Direction Approach}

There are ten subjects who experimented with a scenario of recognizing with a siren sound when an ambulance approached from lateral direction. Only two subjects are able to be considered, others are according to the specific individuality of the subject. As for this scenario, the average data of two persons is shown in Table 3. Figure 9 shows the data of brake depression amount, velocity, deceleration and jerk.

At the time of recognition, the diagonal distance between the target vehicle and the ambulance is $85.7 \mathrm{~m}$, and the velocity of the target vehicle is approximately $50.8 \mathrm{~km} / \mathrm{h}$. The subject recognizes the approach of the ambulance with the siren sound at $53.0 \mathrm{~s}$. After recognition, he starts applying the brake and stops at $59.9 \mathrm{~s}$ and gives way to the ambulance. A maximum deceleration of $-4.76 \mathrm{~m} / \mathrm{s}^{2}$ and a jerk of $-1.26 \mathrm{~m} / \mathrm{s}^{3}$ have occurred until the target vehicle stops.

\section{Safety and Ride Comforts}

In the previous chapter, while vehicle driven by a human traveling on public roads, when it encounters an ambulance, the time of recognizing an ambulance and driver's action are investigated in the case of siren sound and red light. The average data in case of the awareness by siren sound and red light are summarized in Table 3. The scenario of backward 
Table 3 The average data of the subjects notifying an ambulance by siren sound and red light

\begin{tabular}{lllllll}
\hline $\begin{array}{l}\text { Direction of } \\
\text { Ambulance }\end{array}$ & $\begin{array}{l}\text { Recognized } \\
\text { Media }\end{array}$ & $\begin{array}{l}\text { Velocity at } \\
\text { recognition } \\
{[\mathrm{km} / \mathrm{h}]}\end{array}$ & $\begin{array}{l}\text { Distance at } \\
\text { recognition } \\
{[\mathrm{m}]}\end{array}$ & $\begin{array}{l}\text { Time } \\
\text { before stop } \\
{[\mathrm{sec}]}\end{array}$ & $\begin{array}{l}\text { Maximum } \\
\text { declaration } \\
{\left[\mathrm{m} / \mathrm{s}^{2}\right]}\end{array}$ & $\begin{array}{l}\text { Maximum } \\
\text { jerk }\left[\mathrm{m} / \mathrm{s}^{3}\right]\end{array}$ \\
\hline Backward & Siren sound & 30.34 & 56.46 & 9.02 & -2.808 & -0.626 \\
$\begin{array}{l}\text { Backward } \\
\begin{array}{c}\text { Literal } \\
\text { direction }\end{array}\end{array}$ & Siren sound & 40.24 & 53.95 & 11.74 & -3.426 & -0.908 \\
\hline
\end{tabular}

approach and siren sound recognition is the average of five subjects, the average of five subjects as for the backward approach and red light recognition, and the average of two subjects as for the lateral direction and siren sound recognition.

In principle, it should mathematically extract and analyze the deceleration behavior of the subject in each situation for each experimental scenario. The deceleration behavior of a subject varies greatly depending on traffic conditions and emotions, and even if the subject is the same, it is extremely difficult to set up a mathematical model. Therefore, the average data of multiple subjects for each experimental scenario is used. It could be assumed that the referenced data represents the trend of the scheme, not specific data of a specific subject.

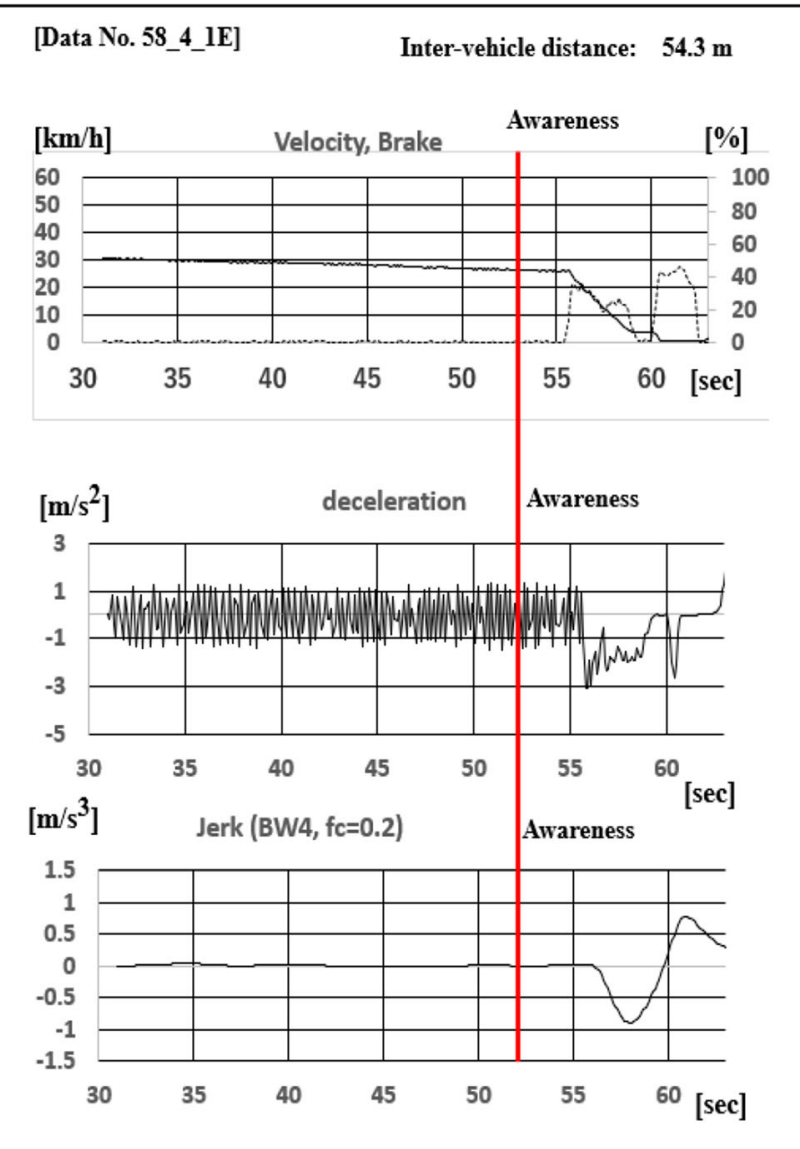

Fig. 8 Velocity \& Brake, Deceleration and Jerk in Notice by red light from backward
Next, authors examine whether the results are acceptable for the safety and comfort of the passenger of automated driving vehicles. There are previous studies on car safety and ride comfort [5]. Safety and ride comfort have been reported to have a major impact on vehicle acceleration and jerk. Especially, dis-comfort is affected by jerk more than the magnitude of the deceleration itself [6].

The safety standards relating to the deceleration and jerk values of automated driving vehicles have not been decided yet. Regarding the current acceleration and deceleration, there is a standard of acceleration determined under the test conditions of the seat belt $[7,8]$, that is " $-2.94 \mathrm{~m} / \mathrm{s}^{2}$ ". Furthermore, the deceleration in sudden braking seems to be instructed to be $-0.3 \mathrm{~g}$ or less at driving schools in Japan. As for jerks, research has been actively conducted on railways. For railway

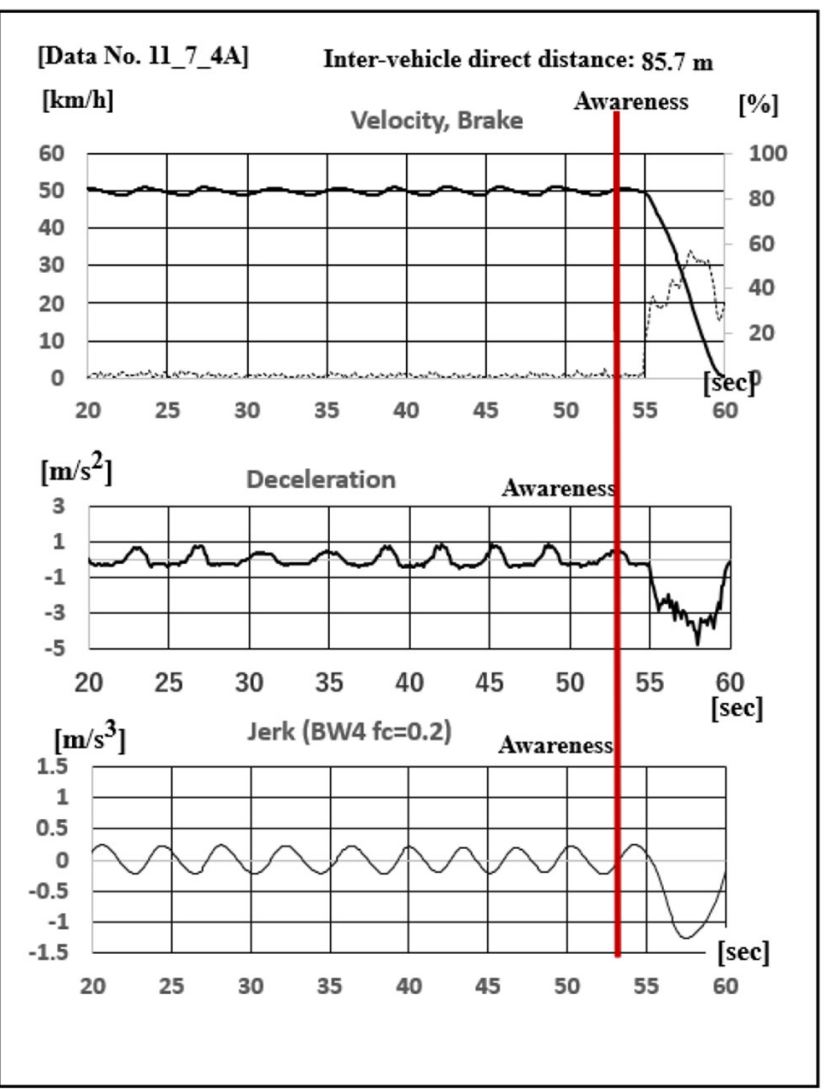

Fig. 9 Velocity \& Brake, Deceleration and Jerk in Notice by siren sound from lateral direction 
vehicle, the steady-state acceleration in the left-right direction is $0.8 \mathrm{~m} / \mathrm{s}^{2}$ or less for the ride when passing a curve. Based on this indication, jerk of $-0.784 \mathrm{~m} / \mathrm{s}^{3}$ for safety brakes and jerk of $-1.25 \mathrm{~m} / \mathrm{s}^{3}$ for emergency brakes are used as important reference values.

The previous study states that the driving style recognized as comfortable for autonomous driving is low jerk and that early action brings out a sense of safety. In our experiments, when there is little time to spare, there is a case where the sense of safety is lost by a short-term action. It is same as the referenced research [6]. However, as for the conclusion that the preferred driving style of automatic driving does not always match the driving method of human drivers, it is necessary to be more studied about the relationship between the driving manner of an expert driver and the preferred automated driving style because of the fact an expert driver not giving stress to passengers. The reference [9] assumes that nonemergency accelerations that can be accepted in public transport are in the range $1.08 \mathrm{~m} / \mathrm{s}^{2}$ to $1.47 \mathrm{~m} / \mathrm{s}^{2}$. These figures are reference examples in public transport and are assumed to be milder than of automobiles. Considering future safety standard, the ride comfort of the automated driving car should be set to be more suitable value. In this paper, authors define “- $2.94 \mathrm{~m} / \mathrm{s}^{2}$ " as the maximum allowable deceleration of a reference value for deceleration and define " $-0.784 \mathrm{~m} / \mathrm{s}^{3 "}$ " as the maximum allowable jerk of a reference value for jerk when discussing the safety of automated driving vehicles.

The data measured in the experiment is usually mixed with various noises, so simply differentiating the deceleration numerically will amplify the noise instead. Therefore, the difference in deceleration is smoothed by the digital filter with a filtering characteristic that is less susceptible to noise and the like. The digital filter used is the Butterworth filter. The coefficients are derived by substituting the parameter of the fourth-order cutoff frequency of $0.2 \mathrm{~Hz}$ into the MATLAB "butter" function. Equation (1) shows the transfer function of Butterworth filter.

$$
\begin{aligned}
H(z) & =\frac{B(z)}{A(z)} \\
& =\frac{b(1)+b(2) z^{-1}+b(3) z^{-2}+b(4) z^{-3}+b(5) z^{-4}}{a(1)+a(2) z^{-1}+a(3) z^{-2}+a(4) z^{-3}+a(5) z^{-4}}
\end{aligned}
$$

The coefficients are as follows.

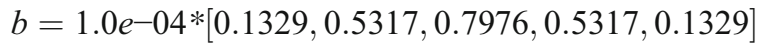

$a=[1.0,-3.6717,5.0680,-3.1160,0.7199]$

When using the maximum allowable deceleration and the maximum allowable jerk defined in this chapter as the evaluation criteria, the experimental data shown in the previous chapter (recognition by siren sound, red light) are barely met in the case of backward and siren sound notification, but other cases are unacceptable.
In addition, even in the case of the backward and siren sound notification, if the time to stop is small, the maximum deceleration exceeds the allowable ground. In summary, the recognized timing being late and the distance and time to stop being small seem to cause a rapid deceleration. Considering how to drive an automated driving vehicle, authors think it is necessary to have an earlier recognition. Chapter 2 describes that vehicle to vehicle communication is advantageous for early recognition. It is effective to recognize early and decelerate in advance when considering the ride comfort of the passengers. In an experiment using a driving simulator, authors closely examine the case where the approach of the ambulance is recognized early by vehicle to vehicle communication and the deceleration is started immediately. The next chapter shows the examples of early recognition of an ambulance by vehicle to vehicle communication and predeceleration.

\section{Experimental Data using V2V Recognition}

\subsection{The case of V2V Recognition from Backward Approach with Pre-Deceleration}

There are five subjects who experimented with a scenario of recognizing with $\mathrm{V} 2 \mathrm{~V}$ communication when an ambulance approached from backward. Only one subject is able to be considered. Others are excluded from the referencing data in this chapter because they are extreme driving behavior such as continuing to run without stopping, or suddenly stopping with emergency brake. Subjects ignore the V2V information or do not notice it. Figure 10 shows the experimental data when a subject recognizes the ambulance approach by vehicle to vehicle communication from backward. The showing data are the brake depression amount, velocity, deceleration and jerk. At the time of recognition, the distance between the target vehicle and the ambulance is $167.4 \mathrm{~m}$, and the velocity of the target vehicle is approximately $28.1 \mathrm{~km} / \mathrm{h}$. The subject recognizes the approach of the ambulance by vehicle to vehicle communication at 39.9 s. After recognition, he starts applying the brake and stops at $52.2 \mathrm{~s}$ and gives way to the ambulance. A maximum deceleration of $-1.94 \mathrm{~m} / \mathrm{s}^{2}$ and a jerk of $-0.41 \mathrm{~m} / \mathrm{s}^{3}$ have occurred until the target vehicle stops. After recognizing the approach of the ambulance, the subject slowly slows down and decelerates in advance. It becomes clear from the interview that siren sound is recognized at $56.4 \mathrm{~s}$.

Thanks to vehicle to vehicle communication, this is the case which recognizes the approach of the ambulance about $16.5 \mathrm{~s}$ earlier than the siren sound. 


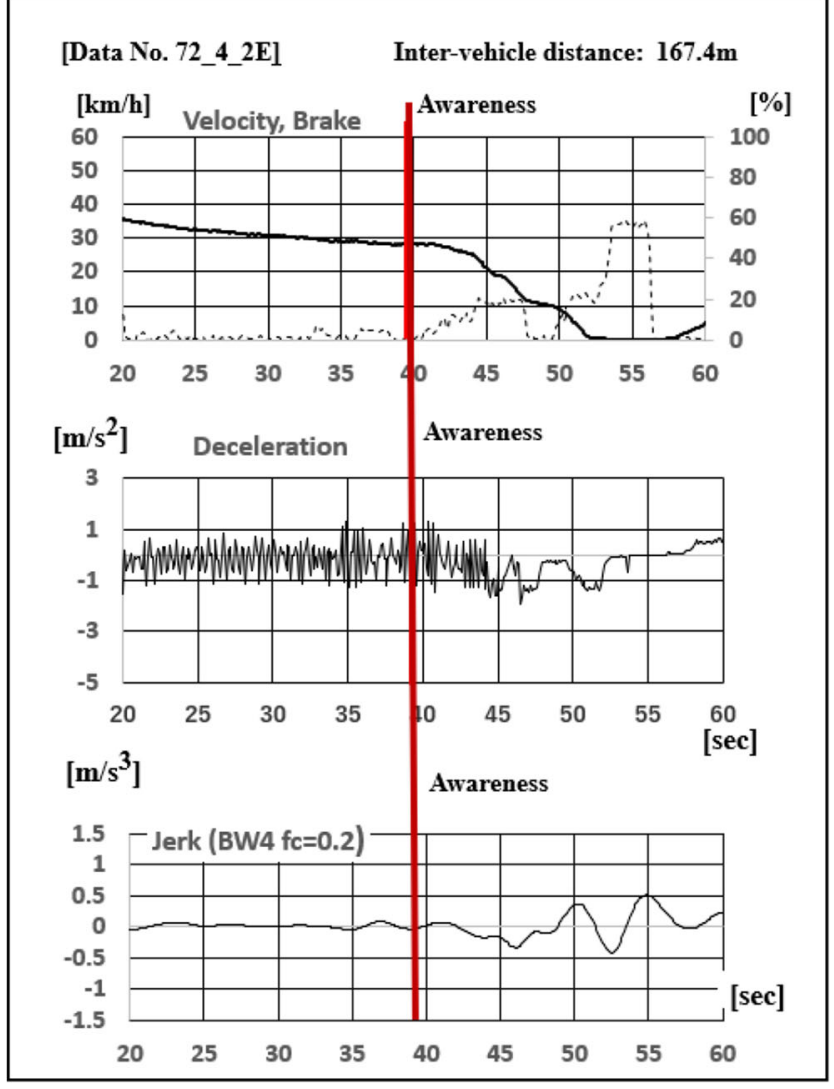

Fig. 10 Velocity \& Brake, Deceleration and Jerk in Notice by V2V from backward

\subsection{The Case of V2V recognition from Lateral Direction Approach with Short Pre-Deceleration}

Figure 11 shows the experimental data when a subject recognizes the ambulance approach by vehicle to vehicle communication from lateral direction. The showing data are the brake depression amount, velocity, deceleration and jerk. At the time of recognition, the diagonal distance between the target vehicle and the ambulance is $121.6 \mathrm{~m}$, and the velocity of the target vehicle is approximately $42.5 \mathrm{~km} / \mathrm{h}$. The subject recognizes the approach of the ambulance by vehicle to vehicle communication at $50 \mathrm{~s}$. After recognition, he starts applying the brake and gives way to the ambulance at $53 \mathrm{~s}$. A maximum deceleration of $-3.77 \mathrm{~m} / \mathrm{s}^{2}$ and a jerk of $-0.67 \mathrm{~m} / \mathrm{s}^{3}$ have occurred until the target vehicle stops. After recognizing the approach of the ambulance, the subject slowly slows down in the constant power and decelerates in advance. It becomes clear from the interview that the time of recognition is $5.0 \mathrm{~s}$ earlier than the siren sound.

It can be seen that both the deceleration and the jerk are smaller compared to the case where the recognition is achieved by siren sound and red light described in the previous section.

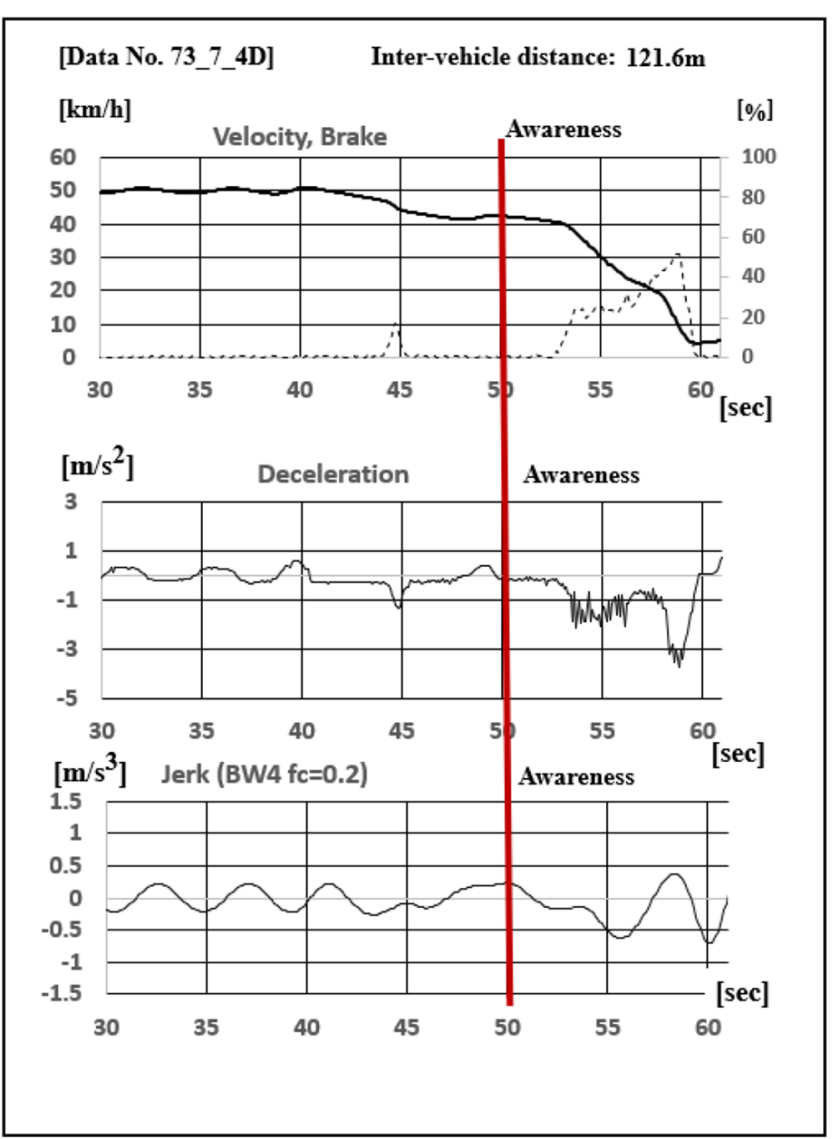

Fig. 11 Velocity \& Brake, Deceleration and Jerk in Notice by V2V from lateral direction

The experimental data in case of the notice by vehicle to vehicle communication are summarized in Table 4 . When the maximum allowable deceleration and the maximum allowable jerk defined in this chapter are used as evaluation criteria, the experimental data by vehicle to vehicle communication becomes an acceptable value except for the deceleration of the approach from the lateral direction. Also in the case of approaching from the lateral direction, the maximum deceleration changes from -4.675 to $-3.77 \mathrm{~m} / \mathrm{s}^{2}$, that is about $0.905 \mathrm{~m} / \mathrm{s}^{2}$ smaller than in the case of recognition from a siren sound.

By simply comparing the data at the time of awareness by siren sound and red light in Table 3. and the data at the time of awareness by vehicle to vehicle communication in Table 4, we could not conclude that vehicle to vehicle communication is a necessary and sufficient condition for acceptable ride comfort. This is because the vehicle slows down slowly in this situation, and comfort can be achieved by slowing down slowly. In order to slow down slowly, other situations other than vehicleto-vehicle communication can be considered (for example, whether there is road information, etc.). However, Tables 3 and 4 show that there is a significant difference in the direct 
Table 4 The data of the subjects notifying an ambulance by V2V

\begin{tabular}{lllllll}
\hline $\begin{array}{l}\text { Direction of } \\
\text { Ambulance }\end{array}$ & $\begin{array}{l}\text { Recognized } \\
\text { Media }\end{array}$ & $\begin{array}{l}\text { Velocity at recognition } \\
{[\mathrm{km} / \mathrm{h}]}\end{array}$ & $\begin{array}{l}\text { Distance at } \\
\text { recognition }[\mathrm{m}]\end{array}$ & $\begin{array}{l}\text { Time before stop } \\
{[\mathrm{sec}]}\end{array}$ & $\begin{array}{l}\text { Maximum declaration } \\
{\left[\mathrm{m} / \mathrm{s}^{2}\right]}\end{array}$ & $\begin{array}{l}\text { Maximum jerk } \\
{\left[\mathrm{m} / \mathrm{s}^{3}\right]}\end{array}$ \\
\hline Backward & V2V & 28.1 & 167.4 & 12.3 & -1.94 & -0.41 \\
Literal direction & V2V & 42.5 & 121.6 & 9.7 & -3.77 & -0.67 \\
\hline
\end{tabular}

distance to the ambulance at the time of awareness between the vehicle to vehicle communication and other means of recognition. For vehicle to vehicle communication, the distance is over $100 \mathrm{~m}$, and the siren and red light are under $73.6 \mathrm{~m}$. This allows vehicle-to-vehicle communication to give the driver time to spare. In other words, vehicle to vehicle communication becomes a sufficient condition for ride comfort.

\section{Adaptation to Automated Driving Vehicles}

On the assumption that an ambulance is encountered on public roads, authors use a driving simulator to check the vehicle's deceleration and jerk before stopping. As a result, in the case of the siren sound and the red light, the deceleration and the jerk are the value above or near to the maximum allowable safety and the maximum allowable jerk defined in this paper.

As the method to recognize an ambulance approach, siren sound and red light are inadequate in the point of the early recognition. Insufficient time results in a sudden slowdown.

If the time to decelerate is earlier, there would be time allowance and the deceleration and jerk would be improved. In order to accelerate the time of deceleration, it is necessary to recognize an early ambulance approach, and the use of vehicle to vehicle communication is desirable.

As a result of experiments in human driving, there is a difference in the deceleration and the jerk on the case with and without the vehicle to vehicle communication. The improvement is seen when the vehicle to vehicle communication is used.

However, it has also been found that the characteristics of the deceleration and the jerk are different depending on the time of starting the pre-deceleration.

Considering adaptation to automated driving vehicle, passenger's safety and ride comfort must be satisfied. The time of starting the pre-deceleration is extremely important and further consideration is needed.

\section{Conclusion and Future Work}

In the absence of guidelines for safety and ride comfort of automated driving vehicles, the authors confirmed the effectiveness of pre-deceleration using vehicle to vehicle communication on assuming maximum allowable deceleration and maximum allowable jerk. On the other hand, vehicle to vehicle communication provides greater recognition distance to emergency vehicles than siren sound and red light. Therefore, there is also a possibility that too early predeceleration occurs. Too early pre-deceleration has a risk of adversely affecting surrounding vehicles. In the future, we would like to study the time to start the optimal predeceleration and create guidelines and standards relating to the safety and ride comfort of automated driving vehicles.

Acknowledgments This study is the results of work supported by Japanese Government Project SIP, the MIC's ICT for the Next Generation ITS [10]. The authors wish to thank the Ministry of Internal Affairs and Communications radio unit mobile communication Division for giving the chance of the study.

Open Access This article is distributed under the terms of the Creative Commons Attribution 4.0 International License (http:// creativecommons.org/licenses/by/4.0/), which permits unrestricted use, distribution, and reproduction in any medium, provided you give appropriate credit to the original author(s) and the source, provide a link to the Creative Commons license, and indicate if changes were made.

\section{References}

1. ASV: Inter-Vehicle Communication (IVC) based Driving Support System. https://www.mlit.go.jp/jidosha/anzen/01asv/resourse/data/ asv3gizyutusetumei.pdf

2. $700 \mathrm{MHz}$ band intelligent transport systems, ARIB standard, ARIB STD-T109. http://www.arib.or.jp/english/html/overview/doc/5STD-T109v1_0-E1.pdf

3. Hideaki, N.A.N.B.A., Yukihito, I.K.A.M.I., Kenichiro, I.M.A.I., Kenji, K.O.B.A.Y.A.S.H.I., Manabu, S.A.W.A.D.A.: An Advantage of the Vehicle to Vehicle Communication for an Automated Driving Car at the Encounter with an Ambulance. IEICE. E101-A(9), 1281-1289 (2018)

4. Hirohiko Baba, Masanao Ebata, "Study on detection of the electronic siren from an ambulance", The Journal of the Acoustical of America 100, 2819 (1996). https://www.asa/scitation.org/doi/abs/ 10.1121/1.41617

5. Bellem, H., Thiel, B., Schrauf, M., Krems, J.F.: Comfort in automated driving: An analysis of preferences for different automated driving styles and their dependence on personality traits. Transport. Res. F: Traffic Psychol. Behav. 55, 90-100 (2018)

6. Hanna Bellem, "Comfort in Automated Driving: Analysis of Driving Style Preference in Automated Driving", Doctor Dissertation, Technische Universitat Chemnitz, Germany, 2018. http://monarch.qucosa.de/api/qucosa\%3A21407/attahment/ATT-0/

7. Japanese Industrial Standards (JIS) D4604, 95th Edition, 2006, "Automotive Parts - Seat Belts", Japanese Standards Association (JSA) 
8. Mohamed Elbanhawi, Milan Simic, Reza Jazar, "In the Passenger Seat: Investigating Ride Comfort Measures in Autonomous Cars," IEEE Intelligent Transportation Systems Magazine, vol.7, no.4, 2015. https://www.researchgate.net/publication/280597370_In_ thePassenger_Seat_Investigating_Ride_Comfort_Measures_ inAutonomous Cars

9. L.L. Hoberock, "A Survey of Longitudinal Acceleration Comfort Studies in Ground Transportation Vehicles", J. Dyn. Sys., Meas., Control 99(2), pp.76-84, 1977. https://repositories.lib.utexas.edu/ handle/2152/20856

10. Hideaki NANBA, "Next-generation ITS to Support Information Communication", New Breeze Vol.27 No.3, 2015 https://www. ituaj.jp/wp-content/uploads/2015/07/nb27-3_web.pdf

Publisher's Note Springer Nature remains neutral with regard to jurisdictional claims in published maps and institutional affiliations.

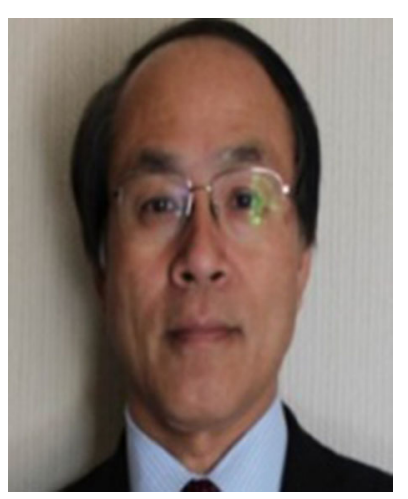

Hideaki Nanba received the B.S. and the M.S. degrees of Information and Computer Sciences from Osaka University in 1977 and 1979, respectively. Since 1979, he works for DENSO CORPORATION as a research engineer. He was one of leaders of the SIP MIC Project, "Development of V2V, V2I Communication Technology Toward the Automated Driving Systems". He is interested in the application of wireless technology to automated driving vehicle.

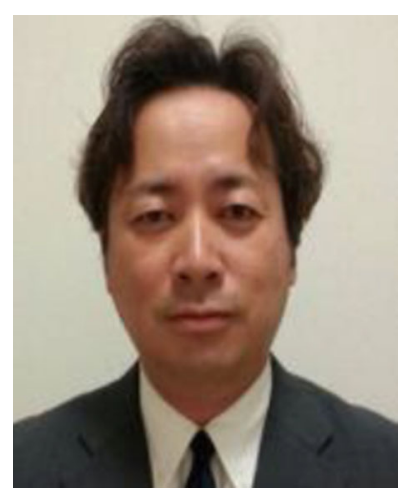

Manabu Sawada received the B.S, M.S. and Ph.D degrees from Nagoya University, Japan in 1992, 1994 and 2007, respectively. In 1994, he joined Research Laboratories, Nippondenso Co., L t d . ( n o w D E N S O CORPORATION). His current research interests include mobile communication systems and ITS. $\mathrm{He}$ is a member of IEEE.

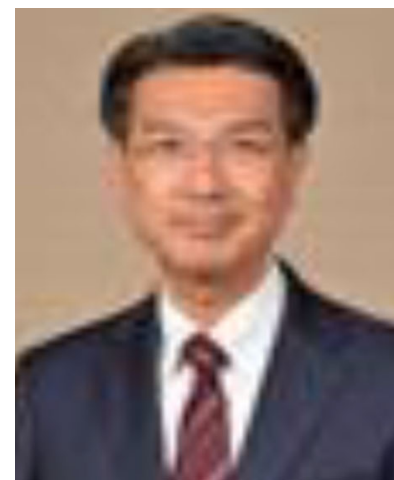

Koji Oguri received the MSc and Ph.D. in Engineering from Nagoya Institute of Technology Japan in 1987 and 1990. He has worked for Nagoya Institute of Technology from 1990 to 1994 as an Assistant Professor, Aichi Prefectural University from 1994 to1998 as an Associate Professor, from 1998 to now as a Professor. He is now a Director of Institute of Information Science and Technology of Aichi Prefectural University. His research interests are in an Intelligent Information Processing and Biomedical Engineering. He is a Member in "IEEE ITS Society", and "IEEE EMB Society". 